\title{
The psychological profile of women presenting to a multidisciplinary clinic for chronic pelvic pain: high levels of psychological dysfunction and implications for practice
}

\author{
This article was published in the following Dove Press journal: \\ Journal of Pain Research \\ 16 November 2016 \\ Number of times this article has been viewed
}

\section{Christina Bryant ${ }^{1,2}$ \\ Rebecca Cockburn' \\ Anne-Florence Plante ${ }^{3}$ \\ Angela $\mathrm{Chia}^{4}$}

'Centre for Women's Mental Health, Royal Women's Hospital, Parkville, ${ }^{2}$ Melbourne School of Psychological Sciences, University of Melbourne, ${ }^{3}$ Department of Physiotherapy, ${ }^{4}$ Department of Anaesthesia, Royal Women's Hospital, Parkville, VIC, Australia
Correspondence: Christina Bryant The Royal Women's Hospital, Locked Bag 300, Cnr Grattan St and Flemington Rd, Parkville VIC 3052, Australia

Email christina.bryant@thewomens.org.au
Objective: Chronic pelvic pain (CPP) is widely acknowledged as a common problem with significant consequences for those diagnosed with this condition. There is a lack of studies with good sample size that provide a comprehensive psychological profile of women presenting to specialist chronic pain clinics. Therefore, the objective of this study was to describe the psychological profile of a representative sample of women presenting with CPP at a tertiary referral center.

Design: This was a cross-sectional study. Women were asked to complete a questionnaire assessing symptoms of anxiety and depression, pain severity and interference, pain self-efficacy and catastrophizing beliefs, and sexual functioning.

Methods: One-hundred and seventy-five women with CPP were recruited when they attended their initial assessment at a specialist CPP clinic of the Royal Women's Hospital, a public hospital in Melbourne, Australia.

Results: Over $75 \%$ of the participants had experienced pain for longer than 2 years. Fiftythree percent of women experienced either moderate or severe anxiety, and $26.7 \%$ experienced moderate-to-severe depression. There were strong correlations between depressive symptoms and pain interference, pain catastrophizing and self-efficacy beliefs.

Conclusion: Our findings confirm previous evidence for high levels of psychological distress and functional impairment associated with this condition, and extend these findings by including measures that are highly relevant to treatment planning, such as thinking styles and pain self-efficacy. Therefore, treatment of this complex condition needs to be holistic, and a multidisciplinary approach is likely to be the best way to achieve this.

Keywords: chronic pelvic pain, women's health, adult, hospital-based clinics, psychological health

\section{Introduction}

Chronic pelvic pain (CPP) is now widely acknowledged as a common problem with significant consequences for those diagnosed with this condition. ${ }^{1}$ Although CPP can affect men and women, this article focuses on pelvic pain in women. Definitions 
of the condition are somewhat imprecise, but typically specify that the pain occurs in the lower abdominal area for at least 6 months, and is unrelated to menstruation, intercourse, or pregnancy. ${ }^{2-4} \mathrm{CPP}$ in women can have many causes that often provide only a partial explanation for the subjective experience reported by the patient. ${ }^{3}$ These causes include endometriosis, pelvic inflammatory disease, and pelvic adhesions. A recent systematic review by Latthe et $\mathrm{al}^{5}$ evaluated more than 60 potential risk factors for pelvic pain, and identified strong and consistent support for three key factors: the presence of pelvic pathology, history of abuse, and comorbid psychological conditions. These three factors have been a consistent focus of research in this area.

Community-based studies estimate the prevalence of CPP to be between $15.8 \%$ and $25 \% .{ }^{6,7}$ Moreover, it is often debilitating, affecting a woman's personal relationships, ability to work, sexual functioning, and self-esteem. ${ }^{8,9}$ In recent years a number of guides to the management of this condition have been published ${ }^{10}$ and increasing clinical attention is being paid to the condition, ${ }^{11}$ with an emphasis on moving away from an organ-specific model of CPP to a broad biospychosocial understanding of this condition. Nevertheless, women often find the experience of clinical assessment and treatment unsatisfactory. ${ }^{12}$ Qualitative studies reveal that many women with CPP feel misunderstood by their medical practitioners, who are perceived to not understand the extent or seriousness of their pain, and gave them the impression that the pain is "all in my head". 9

There are several possible reasons for this, including the inherent complexity of the condition. ${ }^{3}$ Moreover, there are still considerable gaps in our knowledge about the condition, resulting in limited understanding of the profiles of women presenting to pelvic pain clinics. An article on the profile of women with CPP by Reiter ${ }^{13}$ focused on demographic factors and their medical history, rather than their psychological characteristics. Grace and Zondervan ${ }^{2}$ also reported on the physical comorbidities of CPP, stating that $68.7 \%$ of women with CPP had experienced pain other than CPP and/or fatigue in the previous 12 months. Some authors including Ghaly and Chien $^{3}$ and Romao et al have reported on the high prevalence of depression, somatization disorders, and childhood sexual abuse in women with CPP. ${ }^{14}$ Others have questioned whether there is a direct and specific link between childhood sexual abuse and CPP, emphasizing instead the relevance of more recent sexual trauma and the effects of re-victimization on the expression of CPP. ${ }^{15-18}$

Relatively few studies have focused on the psychological profiles of women with CPP. Exceptions include
Thomas et $\mathrm{al}^{18}$ and Weijenborg et al. ${ }^{19}$ The fear-avoidance model of pain, commonly applied to musculoskeletal pain conditions, has also been found to be relevant to women with CPP. ${ }^{20}$ This study highlighted the importance of negative beliefs about the pain ("pain catastrophizing"). A recent study by Miller-Matero et $\mathrm{al}^{21}$ reported on relationships between functioning pain and psychological variables, but this focused only on symptoms of anxiety and depression. A study of illness perceptions in a clinic population highlighted the weak self-efficacy beliefs held by women with CPP, and their perception that their condition was confusing and perplexing. ${ }^{22}$

In general, the articles cited above focus either on broad issues, such as depression, or rather specific psychological aspects of pain (eg, emotion suppression). There is a lack of studies with good sample size that provide a more comprehensive profile of women presenting to specialist chronic pain clinics. A better understanding of the psychological needs of women with CPP is essential for the planning of effective interventions and appropriate services. Therefore, the aim of this study was to address these shortcomings by describing the psychological profile of a representative sample of women presenting with CPP at a tertiary referral center. We used a range of measures to capture pain experience, pain self-efficacy, thinking style, depression, anxiety, and sexual functioning that are important for treatment of this condition. We hypothesized that women attending the clinic would experience high levels of anxiety and depression, and that higher scores on anxiety and depression measures would be associated with higher levels of pain interference, pain catastrophizing, and lower self-efficacy.

\section{Methods}

\section{Setting and participants}

This study employed a questionnaire methodology with participants recruited from the Chronic Pelvic Pain Clinic (CPPC) of the Royal Women's Hospital, a public hospital situated in a metropolitan area of Australia. The clinic has a multidisciplinary team approach in which a pain specialist (consultant anesthetist), clinical psychologist, physiotherapist, and social worker assist women in the treatment and management of their pain. Women were eligible to be included in the study if they were aged 18 years or older, were currently experiencing CPP, and attending the CPPC. Diagnosis of CPP was made by the medical pain specialist in the clinic. From June 2008 to October 2014, 185 consecutive new referrals attended the clinic, of whom $95 \%$ completed the questionnaires, resulting in a sample size of 175 . 


\section{Procedure and measures}

All women attending their initial assessment at the CPPC are asked to complete a series of questionnaires on arrival. The study was approved by the Royal Women's Hospital Ethics Committee. All participants gave written informed consent for their data to be used for research purposes, and then filled out the relevant documents.

The measures were selected with a view to identifying the nature and extent of the women's pain experience, as well as gathering data on a range of psychological factors known to be important to the experience and treatment of chronic pain.

The Brief Pain Inventory (BPI) (Short Form) ${ }^{23}$ is a widelyused measure of pain and its interference with everyday life, comprising two subscales, namely the Pain Severity and Pain Interference Subscales. For the former, participants were asked to rate their current pain intensity as well as their pain in the past 24 hours at its worst, least, and average by indicating on a 10-point Likert scale where $0=$ "no pain" and $10=$ "pain as severe as you can imagine". On the Pain Interference Subscale participants indicated how their pain has affected their quality of life in several different domains (eg, walking, relations with other people) over the past 24 hours (on a 10-point Likert scale where $0=$ "does not interfere" and $10=$ "completely interferes"). The BPI has been found to have acceptable internal consistency (Cronbach's $\alpha$ 0.85) and was able to detect improvement over time in chronic pain patients receiving treatment from a pain-management clinic. ${ }^{23}$

The Hospital Anxiety and Depression Scale (HADS) ${ }^{24}$ was used as a measure of anxiety and depression symptoms. This 14-item scale is used as a screening and severity measure for anxiety and depression in medical outpatients. The HADS has well established psychometric properties (Cronbach's $\alpha 0.80$ ) and was specifically designed for nonpsychiatric medical patients. ${ }^{24} \mathrm{~A}$ score of $0-7$ is considered in the normal range, 8-10 indicates mild anxiety or depression, scores above eleven are indicative of moderate anxiety or depression, and scores $>15$ indicate severe anxiety or depression.

The Pain Self-Efficacy Questionnaire (PSEQ) ${ }^{25}$ was used to ask participants to rate how confidently they could perform a range of listed activities despite their pain. These include items such as household activities, socializing, and coping without medication. Participants answer on a 7-point Likert scale where $0=$ "not at all confident" and $6=$ "completely confident". Scores range from 0-60, with higher scores indicating greater self-efficacy, with a mean score of 25.9 reported in a sample of patients with back pain. The PSEQ has been widely used in studies examining individuals with chronic pain and has been shown to have good internal consistency (Cronbach's $\alpha$ 0.93). ${ }^{26}$

The Coping Strategies Questionnaire (CSQ) ${ }^{27}$ is a 50 -item measure of coping in chronic pain patients. For this study, only the 6-item pain catastrophizing subscale was used. Participants answer on a 7-point Likert scale where $0=$ "never" and 6= "always" in response to items such as, "I feel my life isn't worth living" and "I feel like I can't go on". Thus, total scores can range from $0-36$, with higher scores indicating more dysfunctional attitudes.

The Female Sexual Function Index (FSFI) ${ }^{28}$ is a 36 -item scale assessing six domains of sexual functioning, including desire, orgasm, and pain assessed over the preceding 4 weeks on a 5-point scale. The current study reports data on the interference of pain with vaginal penetration. Participants answered three questions from the original scale: two questions with respect to discomfort of pain during and after penetration, and one assessing the degree of discomfort during or after penetration.

\section{Statistical analysis}

Statistical analysis was carried out using SPSS Version 22 (IBM Corporation, Armonk, NY, USA) to calculate means of all the measures, and correlations between key variables. Composite scores were calculated using the $20 \%$ missing mean replacement rule for the raw item scores on the measures..$^{29}$ Once scaled scores were calculated, a missing values analysis was undertaken, and determined these to be missing at random; therefore the remaining data for composite scores could be imputed using the expectation-maximization technique.

\section{Results}

\section{Pain and interference with functioning}

The mean age of the participants was 36 years (standard deviation $[\mathrm{SD}]=14$ years, range: $18-79$ years). Over $75 \%$ of them had experienced pain for longer than 2 years, and $51.4 \%$ said they could associate a specific event with the pain, ranging from either a past trauma/surgery, to patterns following menstruation, intercourse, etc. The remaining $49.6 \%$ could attribute no causal event to their pain. Around half the women (49\%) deemed their CPP to be the worst pain they had ever experienced compared with a range of other sources of pain, including labor, surgery, and other chronic pains such as back pain and migraines. Scores on the BPI indicated a moderate degree of pain severity, with the mean of composite scores for overall pain severity derived from the four pain severity items being $5.55(\mathrm{SD}=2)$. The mean of composite scores for overall pain interference derived 
Table I Interference of pain with activities

\begin{tabular}{lll}
\hline Item on BPI & $\begin{array}{l}\text { Number of valid } \\
\text { responses }\end{array}$ & M (SD) \\
\hline Pain interfered with general activity & 172 & $5.92(3.066)$ \\
Pain interfered with mood & 173 & $6.34(2.733)$ \\
Pain interfered with walking ability & 173 & $4.76(3.315)$ \\
Pain interfered with normal work & 173 & $6.03(3.25 I)$ \\
Pain interfered with relations with & 172 & $5.48(2.982)$ \\
other people & & $5.86(3.137)$ \\
Pain interfered with sleep & 172 & $6.61(2.923)$ \\
Pain interfered with enjoyment of life & 172 & $5.88(2.970)$ \\
Pain interfered with ability to & 172 & \\
concentrate & & $4.40(3.230)$ \\
Pain interfered with appetite & 173 &
\end{tabular}

Abbreviations: BPI, Brief Pain Inventory; M, mean; SD, standard deviation.

from the nine pain interference items was $5.72(\mathrm{SD}=2.47)$.

Table 1 reports mean interference for each of the activities measured by the BPI, and shows that "enjoyment of life" and "mood" were rated as most interfered with, and had the lowest spread in scores (mean $[\mathrm{M}]=6.61, \mathrm{SD}=2.92 ; \mathrm{M}$ $=6.34, \mathrm{SD}=2.77$, respectively). Interference with "walking ability" and "appetite" received the lowest mean scores, and also the greatest dispersion $(\mathrm{M}=4.76, \mathrm{SD}=3.32 ; \mathrm{M}=4.40$, $\mathrm{SD}=3.23$, respectively).

\section{Symptoms of depression and anxiety}

This group of women experienced significant symptoms of depression and anxiety as measured by the HADS. The mean score on the anxiety scale was $10.82(\mathrm{SD}=4.35)$.

Only $22.9 \%$ of women scored below the cut-off of eight that suggests the presence of mild symptoms. Twenty-four percent experienced mild anxiety (scores ranging from 8-10), $40 \%$ moderate anxiety (11-15), while $13.1 \%$ experienced severe anxiety (16-21). On the HADS depression scale, the mean score was 8.07 ( $\mathrm{SD}=4.45)$, lower than the mean anxiety score and almost half the women (46.9\%) scored below the cut-off of eight. Of those who did endorse symptoms of depression, $26.2 \%$ scored in the mild range, (8-10), $22.3 \%$ in the moderate range (11-15), and $4.6 \%$ experienced severe depression (16-21). There was a significant positive correlation of HADS depression subscale scores with age $(r=0.176, P<0.05)$.

Women in this study reported low levels of pain selfefficacy, with the mean scores on the PSEQ being 23.35. Table 2 gives further details of the item scores.

\section{Pain, coping, and activities}

These show that women were least confident in coping without medication, and had the highest confidence in being
Table 2 Item means on the Pain Self-Efficacy Questionnaire (PSEQ)

\begin{tabular}{|c|c|c|}
\hline Item on PSEQ & $\begin{array}{l}\text { Number of } \\
\text { valid responses }\end{array}$ & $M(S D)$ \\
\hline I can enjoy things, despite the pain & 162 & $2.92(1.642)$ \\
\hline $\begin{array}{l}\text { I can do most of the household chores } \\
\text { despite the pain }\end{array}$ & 162 & 3.18 (1.829) \\
\hline $\begin{array}{l}\text { I can socialize as often as I used to } \\
\text { despite the pain }\end{array}$ & 162 & $2.76(1.972)$ \\
\hline $\begin{array}{l}\text { I can cope with my pain in most } \\
\text { situations }\end{array}$ & 162 & $2.81(1.668)$ \\
\hline $\begin{array}{l}\text { I can do some form of work, despite } \\
\text { the pain }\end{array}$ & 162 & $3.12(1.903)$ \\
\hline $\begin{array}{l}\text { I can still do many of the things I enjoy } \\
\text { doing, despite the pain }\end{array}$ & 162 & 2.54 (1.796) \\
\hline $\begin{array}{l}\text { I can cope with my pain without } \\
\text { medication }\end{array}$ & 162 & $1.63(1.896)$ \\
\hline $\begin{array}{l}\text { I can still accomplish most of my goals } \\
\text { in life, despite the pain }\end{array}$ & 162 & 2.51 (1.856) \\
\hline $\begin{array}{l}\text { I can live a normal lifestyle, despite the } \\
\text { pain }\end{array}$ & 162 & $2.11(1.716)$ \\
\hline $\begin{array}{l}\text { I can gradually become more active, } \\
\text { despite the pain }\end{array}$ & 162 & $2.38(1.762)$ \\
\hline
\end{tabular}

Abbreviations: $M$, mean; SD, standard deviation.

Table 3 Item scores on the catastrophizing subscale of the Coping Strategies Questionnaire (CSQ)

\begin{tabular}{lll}
\hline Individual item in CSQ & $\begin{array}{l}\text { Number of } \\
\text { valid responses }\end{array}$ & M (SD) \\
\hline $\begin{array}{lll}\text { It's terrible and I feel it's never going } \\
\text { to get better }\end{array}$ & 170 & $4.29(I .575)$ \\
It's awful and I feel it overwhelms me & 170 & $4.19(I .589)$ \\
I feel my life isn't worth living & 170 & $2.12(I .858)$ \\
I worry all the time about whether it & 170 & $3.84(I .79 I)$ \\
will end & & $3.99(I .726)$ \\
I feel I can't stand it anymore & $I 70$ & $2.53(I .945)$ \\
I feel like I can't go on & 170 &
\end{tabular}

Abbreviations: $M$, mean; SD, standard deviation.

able to do household chores and doing some form of work. Another measure of beliefs about pain is the catastrophizing subscale of the CSQ, and in this study responses to the CSQ catastrophizing subscale were approximately normally distributed around the mean. The average catastrophizing score was 20.96, with the mean score for individual items being 3.5 ( $\mathrm{SD}=1.4$ ) on a 7-point Likert scale, indicating moderate catastrophizing, but with substantial variance among individuals. Table 3 presents item scores, showing that the lowest mean catastrophizing score was "I feel my life isn't worth living," with a mean response of $2.12(\mathrm{SD}=1.86)$. On the other hand, the mean rating of the item "It's terrible and I feel it's never going to get better" was 4.29 ( $\mathrm{SD}=1.58)$ on a Likert scale, indicating high catastrophizing about the long-term prognosis of their CPP. 
Table 4 Correlation matrix of key study variables

\begin{tabular}{llllll}
\hline Variables & $\mathbf{I}$ & $\mathbf{2}$ & $\mathbf{3}$ & $\mathbf{4}$ & $\mathbf{5}$ \\
\hline I. HADS-A & & & & & \\
2. HADS-D & $0.575^{* *}$ & & & & \\
3. BPI severity & $0.231^{* *}$ & $0.428^{* *}$ & & & \\
4. BPI interference & $0.369^{* *}$ & $0.598^{* *}$ & $0.648^{* *}$ & & \\
5. PSEQ & $-0.212^{* *}$ & $-543^{* *}$ & $-0.296^{* *}$ & $-0.519^{* *}$ & \\
6. CSQ & $0.551^{*}$ & $0.606^{* *}$ & $0.478^{* *}$ & $0.520^{* *}$ & $-485^{* *}$ \\
\hline
\end{tabular}

Notes: *Pearson's correlation is significant at the 0.05 level (two-tailed). **Pearson's correlation is significant at the 0.01 level (two-tailed).

Abbreviations: BPI, Brief Pain Inventory; CSQ, catastrophizing subscale of the Coping Strategies Questionnaire; HADS-A, Hospital Anxiety and Depression Scale anxiety subscale; HADS-D, Hospital Anxiety and Depression Scale depression subscale; PSEQ, Pain Self-Efficacy Questionnaire.

\section{Sexual functioning}

The final measure used to profile the functioning of the participants was the Female Sexual Function Scale (FSFI). Data from this measure showed that in the previous week, $46 \%$ of the sample had not attempted intercourse, and of those who had, $50 \%$ experienced pain or discomfort during vaginal penetration almost always or always, and $75 \%$ experienced pain/discomfort at least half the time. Pain was experienced after penetration almost always or always by $43 \%$ of the women and at least half the time by $72 \%$ of the participants. The discomfort or pain was at least moderate to severe for $83 \%$ of the sample, and high or very high for $58 \%$ of the sample who had attempted intercourse.

\section{Correlations between measures}

We were also interested to know how the observed measures related to each other, and examined this by means of correlational analyses. Pearson's two-tailed correlations were employed. Taking symptoms of depression and anxiety as the primary reference variables we found that scores of the HADS anxiety subscale and HADS depression subscale were significantly correlated with all other key study variables (the BPI, CSQ, and PSEQ). These data are presented in Table 4.

Not surprisingly, the highest correlations were between the anxiety and depression subscales of the HADS (0.606), and between pain severity and pain interference $(0.648)$. Also highly significant were correlations between pain catastrophizing and all other variables.

\section{Discussion}

The aim of this study was to provide a rounded profile of the psychological symptoms and functioning of women attending a tertiary treatment center for CPP. Our findings confirm previous evidence for high levels of psychological distress and functional impairment associated with this condition, ${ }^{21}$ and extend these findings by including measures that are highly relevant to treatment planning, such as thinking styles and pain self-efficacy. Our findings confirm the need to move away from organ-focused approaches to pain, and use integrated models of pain to understand this condition. ${ }^{10,11}$

Consistent with previous work, levels of anxiety symptoms were higher than depressive symptoms, and $53 \%$ of the sample experienced either moderate or severe levels of anxiety symptoms. ${ }^{14,22}$ Anxiety scores on the HADS anxiety subscale were strongly correlated with pain catastrophizing, highlighting the interconnections between cognitive and emotional aspects of pain. Depressive symptoms were less common overall, but more than a quarter of the sample (26.9\%) experienced depressive symptoms in the moderate or severe range. This is lower than the prevalence of $86 \%$ reported by Lorencatto et $\mathrm{al}^{30}$ in a study utilizing the Beck Depression Inventory, but similar to the prevalence of $21 \%$ reported by Poleshuck et $\mathrm{a}^{31}$ and almost identical to the prevalence of $25.7 \%$ reported by Miller-Matero et al, who also used the HADS. ${ }^{21}$ As the HADS was designed for use in hospital outpatient populations it is possible that this provides a more accurate picture than the Beck Depression Inventory, which includes a number of potentially confounding somatic items.

High levels of depression and anxiety are well-established in clinical pain populations, and the reasons for this are complex and varied. ${ }^{32}$ One view is that living with persistent pain and the limitations that this may impose leads to low mood, ${ }^{33}$ but increasing attention is now being paid to biological pathways and neurotransmitters that are shared by pain and depression, such as dopamine in the mesolimbic pathway. ${ }^{34}$ Yet another view proposes that the high prevalence of depression is related to the experiences of childhood neglect, physical and sexual abuse, or domestic violence that are common in the histories of women with CPP. ${ }^{18}$

Perhaps equally important from the clinical point of view is the very strong association between symptoms of depression and a range of key variables known to be associated with greater functional impairment and poorer treatment outcomes. ${ }^{14,33}$ This study found that, depression 
was strongly correlated with lower pain self-efficacy and with catastrophic thinking. These are cross-sectional data, so causality cannot be attributed, but it is highly likely that there is a bi-directional interplay between affective state and pain beliefs, as suggested by Banks and Kerns. ${ }^{35}$ This means that treatment of CPP must address both the symptoms of anxiety and depression and the maladaptive thinking and beliefs with which they are associated.

Indeed, it is concerning that the majority of women in this study reported weak self-efficacy, as holding stronger self-efficacy beliefs is positively associated with use of more active coping behaviors in people with chronic pain. For example, Jensen et al found that self-efficacy beliefs were strongly related to participants' reported coping strategies. ${ }^{36}$ Interestingly, the authors also found that the participants' expectancies regarding coping were only weakly related to actual coping skills and practices, leading them to conclude that it may be more effective for pain management programs to promote behavior change, rather than attitude change. ${ }^{36}$ Although the women in the current study endorsed moderate, rather than severe, levels of catastrophic thinking, there was wide variation in the distribution of scores on this scale, and consistent with prior studies, ${ }^{37}$ a very strong correlation with depression scores was found. This is significant for a number of reasons: experiencing both low mood and negative views of pain and its consequences are likely to pose a double risk for poor outcomes. Depression in those with chronic pain is associated with greater functional impairment and reductions in quality of life, ${ }^{13,38}$ and catastrophic thinking has been linked to poorer outcomes in those with knee osteoarthritis. ${ }^{39}$ Research examining the role of these factors in pelvic pain is lacking, and there is an urgent need for studies to examine these factors prospectively.

Taken together with previous work indicating that women with CPP frequently believe that they have little control over their pain, which they furthermore find perplexing, and believe will be chronic and impairing, ${ }^{22}$ it is clear that the effective treatment of CPP must pay attention to the illness perceptions and beliefs that women hold, which would include a thorough evaluation of illness perceptions, providing education about pain, and, where appropriate, challenging unhelpful or inaccurate perceptions and actively linking this to behavior change. An important aspect of this is to improve self-efficacy and decrease overly negative cognitions. One approach to this is for physiotherapists to empower the patient's bodily experience through techniques such as graded exposure. ${ }^{40}$ Data from other multidisciplinary clinics also highlight the importance of integrating the physical and psychological aspects of treatment, including increasing physical activity. ${ }^{11}$

\section{Strengths and limitations of the study}

One of the strengths of this study was the very high participation rate: $95 \%$ of consecutive referrals to the CPPC agreed to participate. Together with the large sample size of 175 , we can be confident that these data are representative of the women who attend this tertiary clinic. We cannot, of course, extrapolate from these women to community-based, nonclinical samples, nor were we able to compare our sample with individuals with other types of pain. Nevertheless, these findings share some similarities with other studies based on clinical populations, for example, those of Miller-Matero et al. ${ }^{21}$

A further strength of the study was the use of a carefully selected range of measures. These measures are not exhaustive, however - there are aspects of pain we could have made enquiry about, for example, the presence of trauma and revictimization symptoms, which are known to be associated with CPP, ${ }^{18}$ locus of control, emotional suppression, and work role functioning, to name but a few. Our measures were also all based on self-report, which is an intrinsic challenge in assessing subjective experiences, such as pain. In addition, our database did not provide information on other pain experienced by our participants. Nevertheless, these measures provide a more rounded picture of women presenting to a tertiary referral center than has hitherto been available, and is unique in the Australian context.

One important limitation is the cross-sectional nature of our data, which are only available from one time-point. We cannot therefore establish the causal pathways between pain, anxiety, depression, and dysfunctional beliefs. On the basis of previous research, however, we have good reason to believe that they are reciprocal, and need to be taken into account in treatment planning. ${ }^{11,20}$ Future work should seek to follow women up after they have completed treatment in order to ascertain the changes that may or may not result from treatment. We have attempted to do this in our clinic, but had very low rates of retention and data completion.

\section{Conclusion}

We have shown that in a large, representative sample of women attending a tertiary treatment center for CPP participants experienced high levels of anxiety and depression, high levels of pain severity and interference over long periods of time (three quarters of the women in our study had pain for more than 2 years). This was associated with low levels of 
pain self-efficacy and moderate levels of pain catastrophizing. As these are known to be poor prognostic indicators for treatment success, it is essential that clinicians take a broad biopsychosocial approach to the treatment of this condition, and communicate this to patients, in order to engage them in effective management of their pain.

Therefore our CPPC takes a holistic approach, in which assessments of all new referrals include evaluations by a pain specialist, a physiotherapist trained in women's health, and a clinical psychologist. A social worker is also available to provide assistance with psychosocial needs. At a subsequent team meeting we formulate joint treatment plans that take into account women's physical, psychological, and medical needs. These are then implemented collaboratively with the patient.

The importance of taking a multi-disciplinary approach to CPP was also highlighted in other recent studies. ${ }^{11,21}$ These authors found that functional impairment and pain interference were more strongly related to psychiatric symptoms, such as anxiety and depression, than to pain severity. Our findings would support that contention, and their proposal that pain management must include the identification of psychological variables that can influence pain outcomes. In the case of pelvic pain this needs to include sexual and relationship history. ${ }^{17}$ Only when these are taken into account, along with relevant medical and physical histories and symptoms, will patients receive the care that this challenging condition demands.

\section{Acknowledgments}

We thank the women who participated in this study, and the clinic staff who assisted with collecting questionnaires.

\section{Disclosure}

The authors report no conflicts of interest in this work.

\section{References}

1. Abercrombie PD, Learman LA. Providing holistic care for women with chronic pelvic pain. J Obstet Gynecol Neonatal Nurs. 2012;41(5): 668-679.

2. Grace V, Zondervan K. Chronic pelvic pain in women in New Zealand: comparative well-being, comorbidity, and impact on work and other activities. Health Care Women Int. 2006;27(7):585-599.

3. Ghaly AF, Chien PW. Chronic pelvic pain: clinical dilemma or clinician's nightmare. Sex Transm Infect. 2000;76(6):419-425.

4. Weijenborg PT, Greeven A, Dekker FW, Peters AA, Ter Kuile MM. Clinical course of chronic pelvic pain in women. Pain. 2007;132 Suppl 1: S117-S123.

5. Latthe P, Mignini L, Gray R, Hills R, Khan K. Factors predisposing women to chronic pelvic pain: systematic review. BMJ. 2006;332(7544): 749-755.

6. Grace VM, Zondervan KT. Chronic pelvic pain in New Zealand: prevalence, pain severity, diagnoses and use of the health services. Aust NZ J Public Health. 2004;28(4):369-375.
7. Pitts MK, Ferris JA, Smith AMA, Shelley JM, Richters J. Prevalence and correlates of three types of pelvic pain in a nationally representative sample of Australian women. Med J Austr. 2008;189(3):138-143.

8. Strzempko Butt F, Chesla C. Relational patterns of couples living with chronic pelvic pain from endometriosis. Qual Health Res. 2007; 17(5):571-585.

9. Jones G, Jenkinson C, Kennedy S. The impact of endometriosis upon quality of life: a qualitative analysis. JPsychosom Obstet Gynecol. 2004; 25(2):123-133.

10. Engeler DS, Baranowski AP, Dinis-Oliveira P, et al. The 2013 EAU guidelines on chronic pelvic pain: is management of chronic pelvic pain a habit, a philosophy, or a science? 10 years of development. Eur Urol. 2013;64(3):431-439.

11. Twiddy H, Lane N, Chawla R, et al. The development and delivery of a female chronic pelvic pain management programme: a specialised interdisciplinary approach. Br J Pain. 2015;9(4):233-240.

12. Stones RW, Lawrence WT, Selfe SA. Lasting impressions: influence of the initial hospital consultation for chronic pelvic pain on dimensions of patient satisfaction at follow-up. J Psychosom Res. 2006;60(2):163-167.

13. Reiter RC. A profile of women with chronic pelvic pain. Clin Obstet Gynecol. 1990;33(1):130-136.

14. Romao AP, Goraye R, Romao GS, et al. High levels of anxiety and depression have a negative effect on quality of life in women with chronic pelvic pain. Int J Clin Pract. 2009;63(5):707-711.

15. As-Sanie S, Clevenger LA, Geisser ME, Williams DA, Roth RS. History of abuse and its relationship to pain experience and depression in women with chronic pelvic pain. Am JObstet Gynecol. 2014;210(4):317.e1-e8.

16. Chandler HK, Ciccone DS, Raphael KG. Localization of pain and selfreported rape in a female community sample. Pain Med. 2006;7(4):344-352.

17. Meltzer-Brody S, Leserman J, Zolnoun D, Steege J, Green E, Teich A. Trauma and posttraumatic stress disorder in women with chronic pelvic pain. Obstet Gynecol. 2007;109(4):902-908.

18. Thomas E, Moss-Morris R, Faquhar C. Coping with emotions and abuse history in women with chronic pelvic pain. J Psychosom Res. 2006;60(1):109-112.

19. Weijenborg PT, Ter Kuile MM, Gopie JP, Spinhoven P. Predictors of outcome in a cohort of women with chronic pelvic pain - a follow-up study. Eur J Pain. 2009;13(7):769-775.

20. Alappattu MJ, Bishop MD. Psychological factors in chronic pelvic pain in women: relevance and application of the fear-avoidance model of pain. Phys Ther. 2011;91(10):1542-1550.

21. Miller-Matero LR, Saulino C, Clark S, Bugenski M, Eshelman A, Eisenstein $\mathrm{D}$. When treating the pain is not enough: a multidisciplinary approach for chronic pelvic pain. Arch Womens Ment Health. 2016;19(2):349-354.

22. Bryant C, Fitzgibbon O, Chia A. Erratum to 'Illness perceptions and psychological distress in women with Chronic Pelvic Pain' [Journal of Psychosomatic Research (2013) Volume 74, Issue 6, Page 545]. J Psychosom Res. 2014;76(1):88.

23. Tan G, Jensen MP, Thornby JI, Shanti BF. Validation of the Brief Pain Inventory for chronic nonmalignant pain. J Pain. 2004;5(2):133-137.

24. Zigmond AS, Snaith RP. The hospital anxiety and depression scale. Acta Psychiatr Scand. 1983;67(6):361-370.

25. Nicholas MK. The pain self-efficacy questionnaire: Taking pain into account. Eur J Pain. 2007;11(2):153-163.

26. Meredith P, Strong S, Feeney JA. Adult attachment, anxiety and pain self-efficacy as predictors of pain intensity and disability. Pain. 2006;123(1-2):146-154.

27. Rosenstiel AK, Keefe FJ. The use of coping strategies in chronic low back pain patients: relationship to patient characteristics and current adjustment. Pain. 1983;17(1):33-44.

28. Rosen R, Brown C, Heiman J, et al. The Female Sexual Function Index (FSFI): a multidimensional self-report instrument for the assessment of female sexual function. J Sex Marital Ther. 2000;26(2):191-208.

29. Downey RG, King C. Missing data in Likert ratings: A comparison of replacement methods. J Gen Psychol. 1998;125(2):175-191.

30. Lorencatto C, Petta CA, Navarro MJ, Bahamondes L, Matos A. Depression in women with endometriosis with and without chronic pelvic pain. Acta Obstet Gynecol Scand. 2006;85(1):88-92. 
31. Poleshuck EL, Bair MJ, Kroenke K, Watts A, Tu X, Giles DE. Pain and depression in gynecology patients. Psychosomatics: J Consult Liaison Psychiatr. 2009;50(3):270-6.

32. Grace VM. Pitfalls of the medical paradigm in chronic pelvic pain. Baillieres Best Pract Res Clin Obstet Gynaecol. 2000;14(3):525-539.

33. Bair MJ, Robinson RL, Katon W, Kroenke K. Depression and pain comorbidity: a literature review. Arch Intern Med. 2003;163(20): 2433-2445.

34. Leitl MD, Onvani S, Bowers MS, et al. Pain-related depression of the mesolimbic dopamine system in rats: expression, blockade by analgesics, and role of endogenous $\kappa$-opioids. Neuropsychopharmacol. 2014;39(3):614-624.

35. Banks SM, Kerns RD. Explaining high rates of depression in chronic pain: A diathesis-stress framework. Psychological Bulletin. 1996; 119(1):95-110.
36. Jensen MP, Turner JA, Romano JM. Self-efficacy and outcome expectancies: relationship to chronic pain coping strategies and adjustment. Pain. 1991;44(3):263-269.

37. Turner JA, Jensen MP, Romano JM. Do beliefs, coping, and catastrophizing independently predict functioning in patients with chronic pain? Pain. 2000;85(1-2):115-125.

38. Lin $\mathrm{CH}$, Yen YC, Chen MC, Chen CC. Depression and pain impair daily functioning and quality of life in patients with major depressive disorder. J Affect Disord. 2014;166:173-178.

39. Rayahin JE, Chmiel JS, Hayes KW, et al. Factors associated with pain experience outcome in knee osteoarthritis. Arthritis Care Res (Hoboken). 2014;66(12):1828-1835.

40. Sadownik LA, Seal BN, Brotto LA. Provoked vestibulodynia-women's experience of participating in a multidisciplinary vulvodynia program. J Sex Med. 2012;9(4):1086-1093.
Journal of Pain Research

\section{Publish your work in this journal}

The Journal of Pain Research is an international, peer reviewed, open access, online journal that welcomes laboratory and clinical findings in the fields of pain research and the prevention and management of pain. Original research, reviews, symposium reports, hypothesis formation and commentaries are all considered for publication.
Dovepress

The manuscript management system is completely online and includes a very quick and fair peer-review system, which is all easy to use. Visit http://www.dovepress.com/testimonials.php to read real quotes from published authors. 